\title{
Optimizing the Teflon thickness for fast neutron detection using a Ge detector
}

\author{
Sylvian Kahane ${ }^{1}$, and Raymond Moreh $^{2}$ \\ ${ }^{1}$ P. O. Box 1630, 84965, Omer, Israel. E-mail: sylviankahane@gmail.com \\ ${ }^{2}$ Department of Physics, Ben-Gurion University, 84120, Beer-Sheva, Israel . E-mail: moreh@bgu.ac.il \\ The optimum Teflon $\left(\mathrm{C}_{2} \mathrm{~F}_{4}\right)_{n}$ thickness for fast neutron detection through the \\ ${ }^{19} \mathrm{~F}(\mathrm{n}, \alpha){ }^{16} \mathrm{~N}$ reaction was calculated and found to be $\approx 5.0 \mathrm{~cm}$. Here, the $6.13 \mathrm{MeV}$ \\ $\gamma$ ray emitted by ${ }^{16} \mathrm{~N}$ is assumed to be detected by a Ge diode. The geometry of the \\ system is discussed and the $\gamma$ line intensity was found to vary weakly with Teflon thick- \\ ness.
}

\section{Introduction}

Several methods are used in the literature for fast neutron detection. Among those methods are: (1) the detection of protons recoiling from the impinging neutrons [1]. (2) the use of plastic and liquid scintillators [2]. (3) the use of Gd-loaded liquid scintillators [3]. (4) ${ }^{3} \mathrm{He}$ gas-filled detectors can be used for both neutron detection and spectroscopy measurements [4]. (5) Semiconductor-based neutron detectors [5]. In other methods the neutrons are first moderated to thermal velocities then captured using $\mathrm{BF}_{3}$ detectors via the ${ }^{10} \mathrm{~B}(\mathrm{n}, \alpha){ }^{7} \mathrm{Li}$ reaction [6]. In addition, fast neutron detection often relies on neutron induced nuclear reaction. The topic of the present work is the use the ${ }^{19} \mathrm{~F}(\mathrm{n}, \alpha){ }^{16} \mathrm{~N}$ reaction [7] to detect fast neutrons with energies $E_{n}>3 \mathrm{MeV}$. This may be done by holding Teflon $\left(\mathrm{C}_{2} \mathrm{~F}_{4}\right)_{n}$ in close vicinity to a Ge gamma detector. When the Teflon is hit by fast neutrons it forms ${ }^{16} \mathrm{~N}$; it is a $\beta$ emitter $(\tau=7.2 \mathrm{~s})$ proceeding to an excited state in ${ }^{16} \mathrm{O}(68 \%)$ which emits a $6.13 \mathrm{MeV}$ photon. This can readily be measured using a Ge detector. Teflon is a combination of $24.0 \%$ $\mathrm{C}$ and $76.0 \% \mathrm{~F}$ (by weight), with a density of $2.2 \mathrm{gm} / \mathrm{cc}$ [8]. Note that because of the high gamma energy emitted by ${ }^{16} \mathrm{O}$, it is easily visible above background and may be viewed as an excellent finger print of fast neutrons. The ${ }^{19} \mathrm{~F}(\mathrm{n}, \alpha){ }^{16} \mathrm{~N}$ reaction is endothermic with $Q=-1.52 \mathrm{MeV}$ and because of the Coulomb barrier viewed by the emitted $\alpha$-particles, a non-zero yield is obtained only for $E_{n}>3 \mathrm{MeV}$. In the past, this reaction was discussed in some detail for the detection of fast neutrons [7] where a Teflon cup covering a $30 \mathrm{cc} \mathrm{Ge}(\mathrm{Li})$ diode was used to detect the $6.13 \mathrm{MeV}$ photon. Our interest here is to calculate the optimum thickness of the Teflon covering a pure $\mathrm{Ge}$ detector.

We use the simple geometry described in Fig.1. The present calculation includes two representative Ge detector volumes: $100 \mathrm{cc}$, and 300 cc. In Fig. 1 the neutron beam is assumed to be monoenergetic with $\mathrm{E}_{n}=5$ to $11 \mathrm{MeV}$, hitting the Teflon in a normal direction (shown by the arrows), or embedded in a neutron field of uniform flux. Results were obtained also for a fission neutron spectrum having a Watt shape.

\section{Simulations}

The goal of the simulations is to "measure" the response of a Ge detector to the gamma rays induced by incoming neutrons on a Teflon

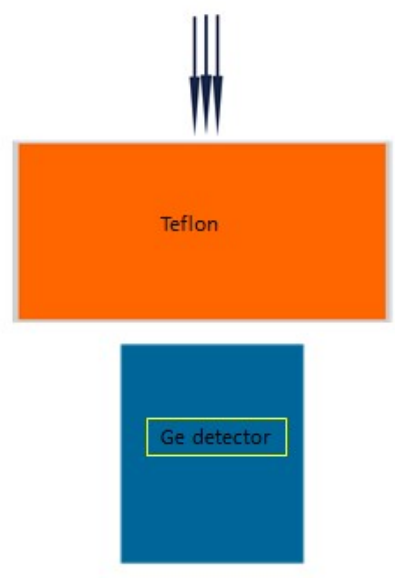

Fig. 1: A pencil neutron beam is hitting few $\mathrm{cm}$ thick Teflon absorber placed in a $1 \mathrm{~mm}$ thick Aluminum case (not shown), at $5.0 \mathrm{~mm}$ above a $\phi 64 \times 90 \mathrm{~mm}$ Ge coaxial detector.

shield, placed in a Aluminum cover $5 \mathrm{~mm}$ above the detector, Fig.1. This is calculated as a function of the Teflon thickness. We are especially interested in the $\beta$ decaying ${ }^{16} \mathrm{~N}$ nuclei proceeding to the excited level in ${ }^{16} \mathrm{O}$ emitting the $6.13 \mathrm{MeV} \gamma$ line. The incoming neutron undergoes nuclear reactions with the Fluorine nuclei producing ${ }^{16} \mathrm{~N}$ by ${ }^{19} \mathrm{~F}(\mathrm{n}, \alpha)$ and ${ }^{15} \mathrm{~N}$ by ${ }^{19} \mathrm{~F}(\mathrm{n}, \alpha+\mathrm{n})$ respectively. ${ }^{15} \mathrm{~N}$ is stable with no further decays or $\gamma$ rays. The respective cross sections, from Janis [9], are shown in Fig.2.

It can be seen that the first reaction has a non-zero cross section at a threshold of $3 \mathrm{MeV}$ while the threshold of the second is $5 \mathrm{MeV}$. The simulations proceed in two steps, one for neutrons and one for gammas. The neutron simulation "measures" the production yield of the ${ }^{16} \mathrm{~N}$ nuclei in Teflon cylinders of different thicknesses. The gamma simulations "measure" the actual detector response to the 6.13 MeV $\gamma$ produced in the same Teflon cylinders. A convolution of the two results produces the response of the detector, per neutron, as a function of the Teflon thickness.

\subsection{Neutrons}

Two different geometries were employed: in one a monoenergetic and monodirectional pencil beam of neutrons impinges on a cylindrical Teflon sheet placed above the Ge detector, Fig.1; in the second, the same Teflon cylinder, is placed in a "bath" of monoenergetic 


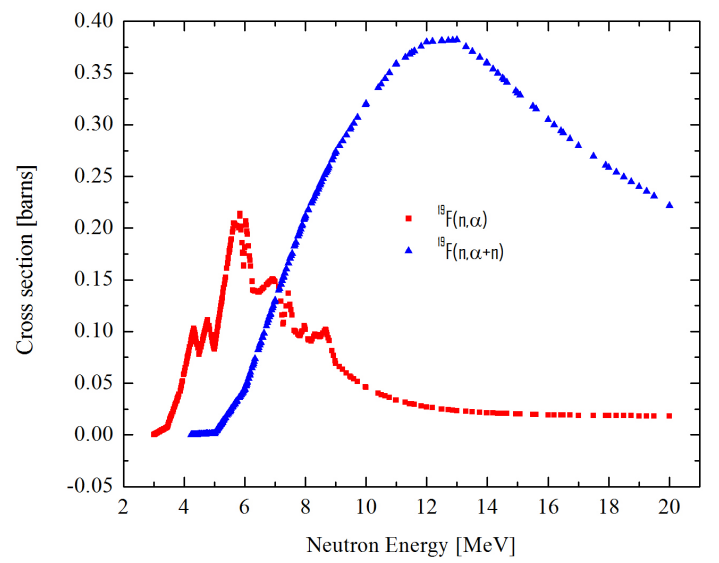

Fig. 2: The cross sections of the ${ }^{19} \mathrm{~F}(\mathrm{n}, \alpha)$ and ${ }^{19} \mathrm{~F}(\mathrm{n}, \alpha+\mathrm{n})$ reactions taken from the Japanese cross sections library JENDL-4.0.

neutrons, simulating a uniform neutron field. The number of ${ }^{16} \mathrm{~N} \mathrm{nu}-$ clei produced is counted and normalized to the number of neutrons used in the simulation. For the present purpose this quantity is called Yield $-{ }^{16} N$ which is the $\gamma$-source of interest. It increases with Teflon thickness reaching a saturation which depends on the extent of neutron absorption (Fig.3). The statistical error in this Monte Carlo calculations is less than $1 \%$, using $10^{6}$ neutrons for the case of a pencil beam and $4 \times 10^{6}$ for an uniform flux of neutrons.

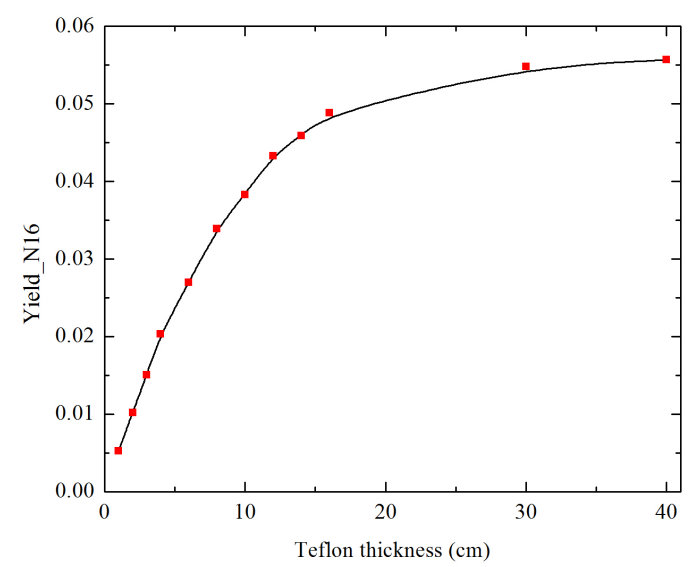

Fig. 3: Calculated yield of ${ }^{16} \mathrm{~N}$ nuclei as a function of Teflon thickness obtained by assuming a neutron pencil beam of $\mathrm{E}_{n}=5 \mathrm{MeV}$. The line is only a guide to the eye.

Additionally, we calculated the distribution of the ${ }^{16} \mathrm{~N}$ nuclei along the $z$ axis of the Teflon cylinders (taken to be along the direction of the normal). Obviously, in the case of a uniform neutron field this distribution is also uniform, but in the case of a pencil beam the nuclei density is highest at the beam entrance, Fig.4.

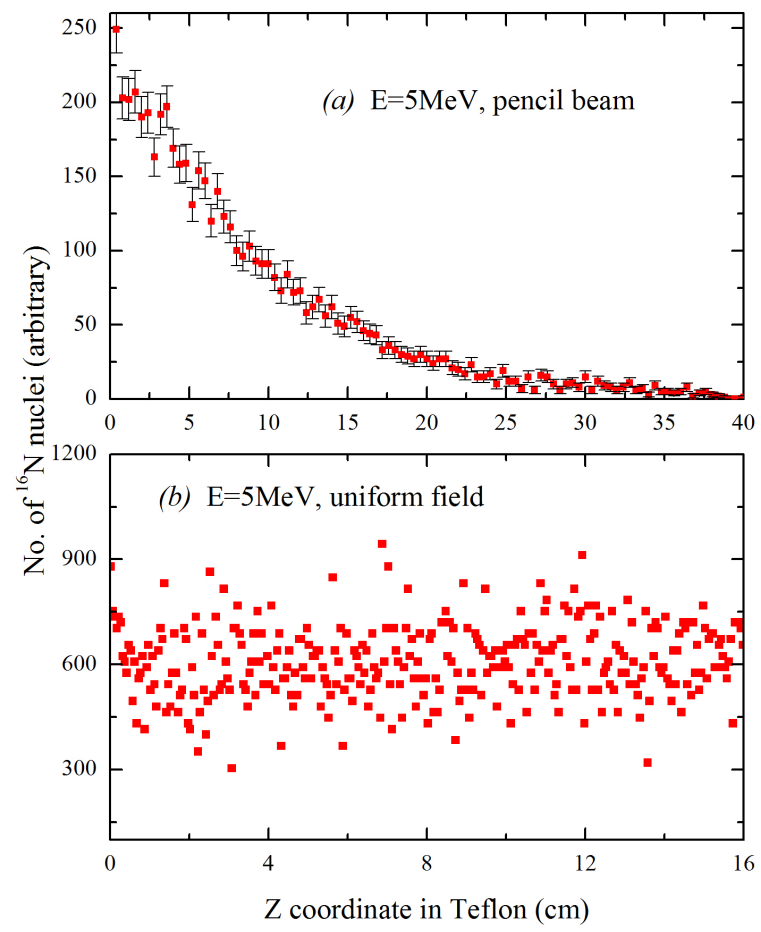

Fig. 4: The distribution of the ${ }^{16} \mathrm{~N}$ nuclei along the $z$ axis of the Teflon cylinder for two cases: (a) pencil beam and (b) uniform flux. In the second case the standard deviation is larger (17\%) but the distribution is undoubtedly uniform.

\subsection{Technical details}

The neutron simulations were performed with Geant4 [10]. This platform was chosen because it produces a plethora of ions in Teflon, both by nuclear reactions and by radioactive decay. An example is given in Table1.

The kinetic energies of the $C$ - and $F$-ions appearing in the table are acquired via elastic and inelastic neutron scattering. The number of $\alpha$ 's is equal to the sum of ${ }^{15} \mathrm{~N}$ and ${ }^{16} \mathrm{~N}$ ions. The total number of gammas $\left(1.4 \times 10^{6}\right)$ is far larger than the ones at $6.13 \mathrm{MeV}\left(4 \times 10^{4}\right)$, but most of the gammas have low energies $<0.3 \mathrm{MeV}$ (Fig.5) and do not interfere with the measurements. The Geant 4 system offers many options concerning the exact physics to be used in the simulations. We borrowed the detailed physics which appears in the example Hadr06 (/examples/extended/hadronic) found in its distribution. It employs high precision (HP) neutron physics i.e. uses actual neutron cross sections, for neutrons under $20 \mathrm{MeV}$, and not models, standard electromagnetic physics, radioactive decay and ion physics based on the internal models used by Geant 4 . Furthermore, the new neutron cross sections, developed by Mendoza and Cano-Ott [11], based on ENDF-VII, were adopted. 
Table 1: Number of ions produced in Teflon by a uniform neutron field of $5 \mathrm{MeV}\left(4 \times 10^{6}\right.$ neutrons). The numbers in square brackets are the energies of excited levels in keV, i.e. ${ }^{19} \mathrm{~F}$ [1554.0] stands for the $1554.0 \mathrm{keV}$ level of ${ }^{19} \mathrm{~F}$.

\begin{tabular}{|l|r|r|}
\hline \multicolumn{1}{|c|}{ Ion } & Numbers produced & Mean Energy \\
\hline${ }^{12} \mathrm{C}$ & 559603 & $436.0 \mathrm{keV}$ \\
${ }^{13} \mathrm{C}$ & 5907 & $404.1 \mathrm{keV}$ \\
${ }^{19} \mathrm{~F}$ & 1414198 & $267.8 \mathrm{keV}$ \\
${ }^{19} \mathrm{~F}[109.9]$ & 46 & $60.9 \mathrm{eV}$ \\
${ }^{19} \mathrm{~F}[1554.0]$ & 1033 & $217.1 \mathrm{eV}$ \\
${ }^{19} \mathrm{~F}[197.1]$ & 1884 & $224.2 \mathrm{eV}$ \\
${ }^{19} \mathrm{~F}[2779.9]$ & 1 & $72.1 \mathrm{eV}$ \\
${ }^{19} \mathrm{~F}[4377.7]$ & 1 & $11.2 \mathrm{eV}$ \\
${ }^{20} \mathrm{~F}$ & 77 & $188.3 \mathrm{keV}$ \\
${ }^{15} \mathrm{~N}$ & 294 & $535.9 \mathrm{keV}$ \\
${ }^{16} \mathrm{~N}$ & 60366 & $891.2 \mathrm{keV}$ \\
${ }^{20} \mathrm{Ne}$ & 77 & $71.7 \mathrm{eV}$ \\
${ }^{20} \mathrm{Ne}[1633.7]$ & 77 & $487.1 \mathrm{eV}$ \\
${ }^{16} \mathrm{O}$ & 60364 & $1.6 \mathrm{keV}$ \\
${ }^{16} \mathrm{O}[6049.4]$ & 9 & $387.2 \mathrm{eV}$ \\
${ }^{16} \mathrm{O}[6129.9]$ & 40378 & $427.6 \mathrm{eV}$ \\
${ }^{16} \mathrm{O}[6917.1]$ & 22 & $128.20 \mathrm{eV}$ \\
${ }^{16} \mathrm{O}[7116.9]$ & 3037 & $260.8 \mathrm{eV}$ \\
${ }^{16} \mathrm{O}[8871.9]$ & 614 & $70.9 \mathrm{eV}$ \\
${ }^{19} \mathrm{O}$ & 2025 & $294.2 \mathrm{keV}$ \\
$\alpha$ & 60660 & $2.38 \mathrm{MeV}$ \\
${ }^{10}$ & 62466 & $3.14 \mathrm{MeV}$ \\
$\mathrm{e}+$ & 2786 & $2.00 \mathrm{MeV}$ \\
$\mathrm{e}-$ & 1416604 & $707.0 \mathrm{keV}$ \\
$\gamma$ & 564213 & $2.72 \mathrm{MeV}$ \\
$\mathrm{Neutron}$ & 2025 & $673.2 \mathrm{keV}$ \\
$\mathrm{Proton}$ & & \\
\hline
\end{tabular}

\subsection{Gammas}

Here, the Teflon cylinder acts as a volume source. The exact departing point of each gamma is sampled in this volume, uniformly in the radial direction, and according to the distributions of Fig.4 in the $z$ direction. The statistical error is negligible. The simulations were carried out for two detector volumes: $100 \mathrm{cc}(\phi 50 \times 51 \mathrm{~mm})$, and $300 \mathrm{cc}(\phi 64 \mathrm{x} 90 \mathrm{~mm})$. These dimensions correspond to one of our detectors $(100 \mathrm{cc})$ or taken from the ORTEC catalog $(300 \mathrm{cc})$. The spectrum of the energy deposition is calculated by assuming no broadening, i.e. with zero energy resolution, in $1 \mathrm{keV}$ bins (Fig.6). This is because we did not compare to an actual measured spectrum but are interested only in the relative peak intensities.

The peak intensities are normalized per one gamma at source. As a function of the Teflon thickness it is a descending plot (less Teflon, less absorption) - Fig.7. In order to obtain the intensities per neutron one has to multiply by the number of gammas found at a given Teflon thickness, this is what we called Yield $-{ }^{16} N$, in Fig.3. One of the graphs is going up (Fig.3) and one is going down (Fig.7), hence a maximum appears at a point corresponding to the optimum thickness - Fig.8.

We sought the optimum with a resolution of $1 \mathrm{~cm}$. We obtained a

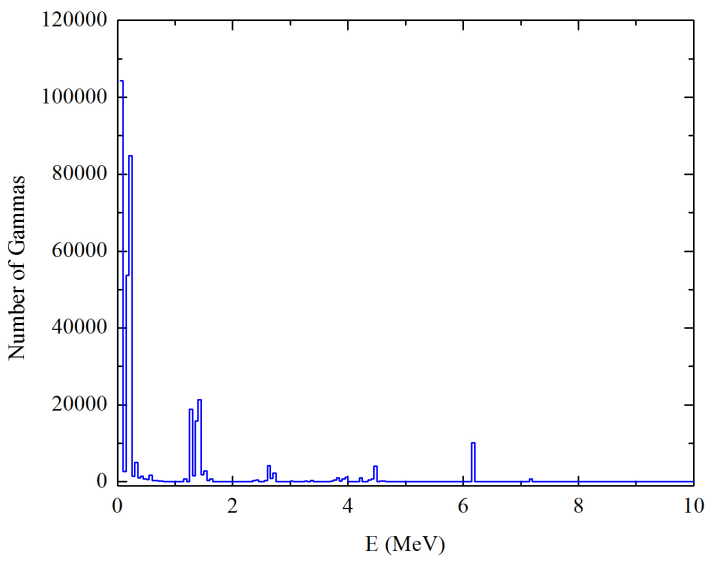

Fig. 5: Energies of the gammas produced in Teflon by $5 \mathrm{MeV}$ neutrons. The gammas at $6.13 \mathrm{MeV}$ are free of any interference.

thickness of $5 \mathrm{~cm}$ for this optimum, for both detectors, for both neutron fields and for all the energies studied (between 5 to $11 \mathrm{MeV}$ ).

Fig. 8 presents the obtained intensities as percentage points where the optimum is $100 \%$. Data come from the first escape (FE) peak in the case of the $100 \mathrm{cc}$ detector and from the photopeak in the case of the $300 \mathrm{cc}$ detector. While the optimum is well defined it is not very sharp, Fig. 8 shows that there are additional values, for the Teflon thickness, which differ from the optimum by only few percent. An interesting point in the results of the calculations is that the optimum thickness is sensitive neither to the incident neutron energy (in the energy range of our calculations) nor to the size of the detector. It may be seen that by varying the Teflon thickness between 4 to $6 \mathrm{~cm}$, the counting rate of the detector varies by few percent only. In general, it can be said that the range $4-6 \mathrm{~cm}$ for Teflon will provide equally good counting results in an actual measurement. Even when using a much thinner Teflon of $2 \mathrm{~cm}$ we are within $15 \%$ from the optimum (in the uniform field case).

\subsection{Fission like neutron spectrum}

In the vicinity of nuclear reactors or accelerators there are non monoenergetic neutron fields. For nuclear reactors one can assume a fission like uniform Watt spectrum:

$$
f(E)=\exp (-E / 0.965) \times \sinh (2.29 \times E)
$$

With the parameters taken from the defaults given in the MCNP manual [12] (the units are $\mathrm{MeV}$ for the first parameter and $\mathrm{MeV}^{-1}$ for the second). Obviously, because we obtained a flat value of 5 $\mathrm{cm}$ for all the energies of interest in the Watt spectrum, the optimum value for a reactor spectrum will be also $5 \mathrm{~cm}$.

\subsection{Other details}

The simulations for the gammas were done with the MCNP program [12]. In principle, they can be done also by using Geant4 but with greater effort. Geant 4 is a library and the user has to possess considerable programming skills in order to build a running pro- 


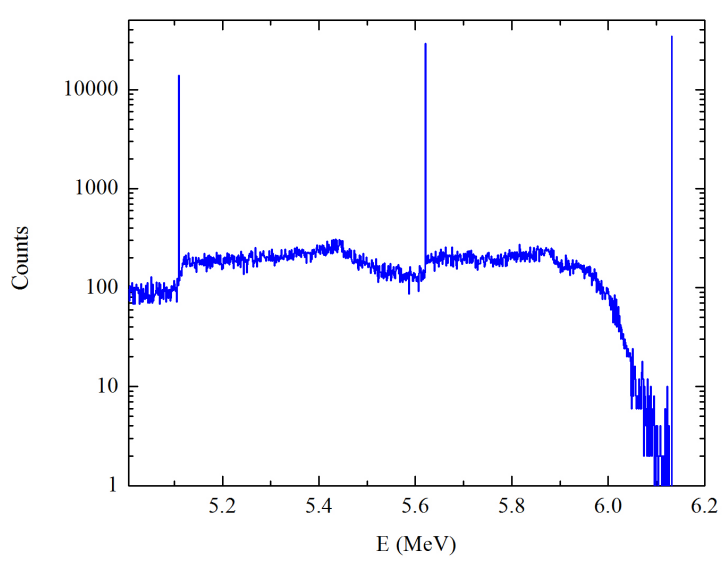

Fig. 6: Calculated spectrum in the 300cc detector from a Teflon shield of $5 \mathrm{~cm}$.

gram. MCNP is a closed, tested program and the user has to provide only the input data.

\section{Conclusions}

As may be seen from the above, the Teflon thickness yielding the optimum intensity of the $6.13 \mathrm{MeV} \gamma$ line is $\approx 5.0 \mathrm{~cm}$. It is surprising to see that this thickness is almost independent on the volume of the Ge detector, on the incident neutron energy (in the range studied) and on the direction of incidence of the neutrons.

\section{Acknowledgements}

One of us (R.M) would like to thank Y. Ben-Galim for help in the initial stages of this work.

Submitted on Month Day, Year / Accepted on Month Day, Year

\section{References}

1. Shultis J.K., McGregor D.S. Efficiencies of coated and perforated semiconductor neutron detectors. IEEE Trans. Nucl. Sci., 2006, v. 53, 1659.

2. Knoll G. F. Radiation Detection and Measurement. John Wiley \& Sons, New York, 2010.

3. Kapoor S. S., Ramamurthy V. S. Nuclear Radiation Detectors. New Age International, New Delhi, 1986.

4. Tsoulfanidis N., Landsberger S. Measurement and Detection of Radiation, 4th Edition. CRC Press, Boca Raton, 2015.

5. Hussein E. M. Handbook on Radiation Probing, Gauging, Imaging and Analysis: Volume I. Kluwer Academic Publishers, New York, 2004.

6. Ben-Galim Y., Wengrowicz U., Moreh R., Orion I., Raveh A., Using the Doppler broadened $\gamma$ line of the ${ }^{10} \mathrm{~B}(\mathrm{n}, \alpha \gamma)^{7} \mathrm{Li}$ reaction for thermal neutron detection. Nucl. Inst. Meth. Phys. Res. A, 2016, v. 810, 140-143.

7. Wolf A., Moreh R., Utilization of Teflon covered Ge(Li) diodes for fast neutrons detection. Nucl. Inst. Meth., 1978, v. 148, 195-197.

8. http://physics.nist.gov/cgi-bin/Star/compos.pl?matno=227.

9. https://www.oecd-nea.org/janis.

10. Agostinelli S. et al. Geant 4 - a simulation toolkit. Nuc. Inst. Meth. Phys. Res. A, 2003, v. 506, 250-303.

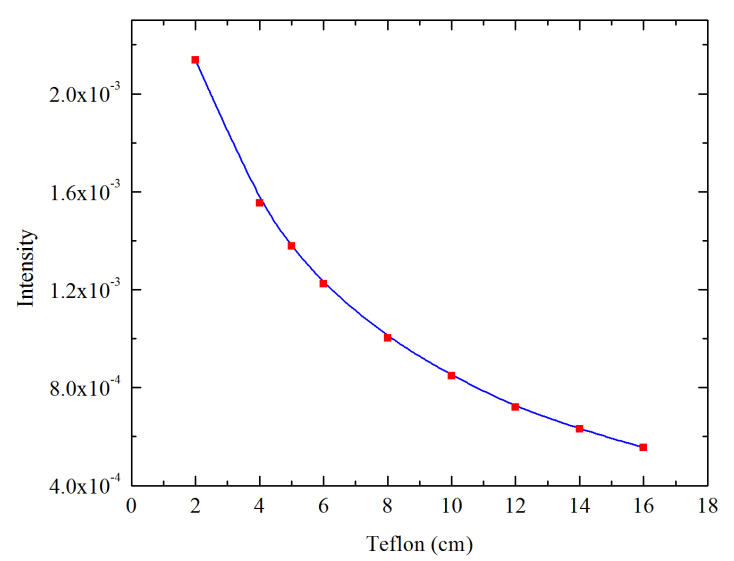

Fig. 7: Photopeak (6.13 MeV) intensity as a function of the Teflon thickness, calculated for the $100 \mathrm{cc}$ detector.

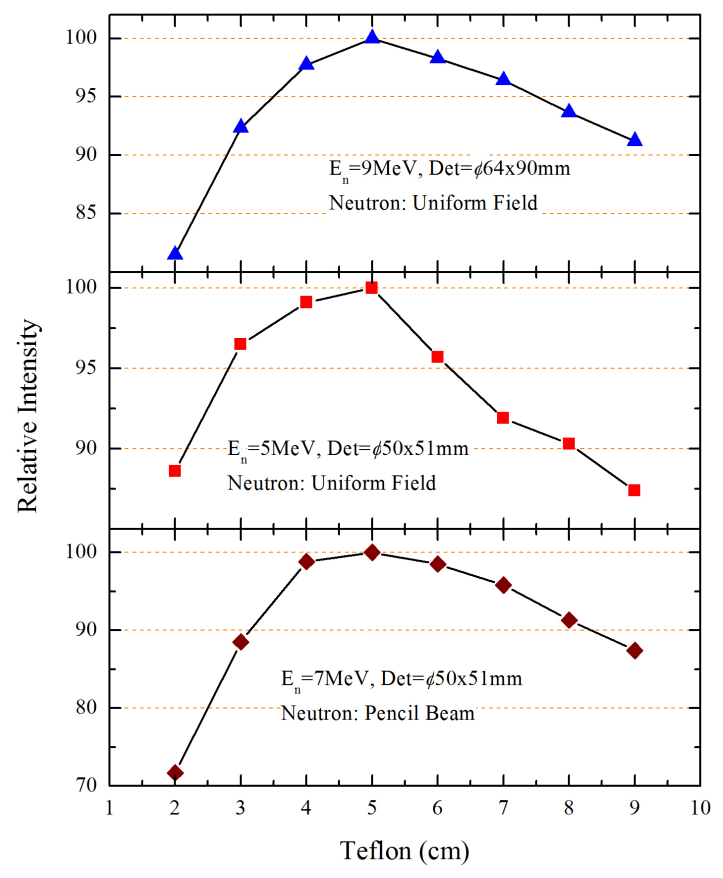

Fig. 8: Relative intensities versus Teflon thickness for various neutron energies and different Ge volumes. Some input data are listed in the figures.

11. https://www-nds.iaea.org/geant4.

12. Briesmeister J.F. MCNP-A General Monte Carlo N-Particle Transport Code. Technical Report LA-13 709-M, Los Alamos National Laboratory, NM, USA, 2001.

Sylvian Kahane and Raymond Moreh Optimizing Teflon ... 